\title{
The drive (D) properties of reproduction-set in a word association task
}

SHLOMO BREZNITZ

THE HEBREW UNIVERSITY, JERUSALEM

Ss who received a deliberate set to reproduce their original responses to a word association test succeeded in reproduction more than control $S$ s when their original responses were "strong." When the original responses were "weak," however, reproduction-set was detrimental to reproduction. The same effect was obtained with latencies of reproductions. These results were analyzed in terms of the Hullian equation in which drive (D) multiplies the response strength of the entire habit family hierarchy.

When testing reproduction (RP) in a word association test in the clinical-diagnostic context, Ss are explicitly required to reproduce their initial responses (e.g., Rapaport, Gill, \& Schafer, 1946), while in studies of the stability of response hierarchies there is no such RP-set (e.g., Moran, Mefferd, \& Kimble, 1964). This lack of standard procedure makes it difficult to evaluate the existing knowledge concerning RP. The main purpose of this study was to test the influence of RPset upon the behavior in a word association task.

One possible way to approach this problem would be to view the specific RP-set as a manipulation that augments the total drive level (D) of S. On the basis of Hull's (1952) equation concerning reaction potential, one should predict that the effect of high $D$ would depend upon the particular position of a response within the habit family hierarchy. Responses which are "strong" (high in the hierarchy) should become even stronger, while "weak" response (low in the hierarchy) should become weaker stlll (e.g., Spence, Farber, \& McFann, 1956; Taylor \& Spence, 1952).

A crucial issue in most of the relevant studles is the determination of strong and weak (or difficult and easy) responses. A frequent procedure is to establish these differences on an a priori analysis of the particular responses studied. Sometimes this is done on the basis of information concerning performance of groups of individuals, and only rarely can the difficulty of a task for each individual be ascertained.

When dealing with a RP task, however, we have at our disposal a slightly different method. It is possible to use some indices of reaction potential, and analyze the original response which would have to be reproduced. For any $S$ then, every response can be categorized as strong or weak on an empirical basis according to the available indices. In the case of a word association test the latency (reaction time) of the flrst response is such an index. The probability and latency of successful reproductions may then be used as convenient dependent variables.
On the basis of this analysis the following three hypotheses may be formulated: (1) The probability of successful reproduction of strong original responses should be greater in the reproduction-set condition. (2) The probability of successful reproduction of weak original responses should be greater in the no-set condition. (3) The latencies of successful reproduction responses should be shorter in the reproduction-set condition.

\section{Subjects and Procedure}

Twenty Ss were in the reproduction-set group, and 30 in the no-set group. All of the $50 \mathrm{Ss}$ (undergraduate volunteers) were tested individually. The word association test was administered with the usual instructions to give the first response to each stimulus word. Immediately following the first test $\left(T_{1}\right)$, the same stimuli were again presented $\left(\mathrm{T}_{2}\right)$. In the reproductionset conditions Ss were requested to reproduce their original responses, whereas in the no-set condition the instructions of $T_{1}$ were repeated. $E$ used a stopwatch to measure the reaction times on both tests. Stimuli

Altogether 20 stimuli were used. The following is the translation from Hebrew of the stimulus words in their order of presentation: tree, fear, picture, sin, flower, chair, morality, concentration, words, bird, worries, paper, lie, loneliness, song, consclence, excitement, apprehensions, stone, punishment.

\section{Results}

Operational definitions of response strength and reproduction. Responses with reaction time on $T_{1}\left(R_{1}\right)$ up to $3 \mathrm{sec}$ were defined as strong responses, and responses with $R t_{1}$ above 3 sec were defined as weak. A response on $T_{2}$ was defined as a reproduction ( $\left.R p+\right)$ if and only if it was exactly identical to the response given to the same stimulus on $\mathrm{T}_{1}$. If that were not the case, it was defined as a reproduction fallure (Rp-).

On the basis of these operational definitions, it is now possible to test the three research hypotheses that have been formulated. Table 1 presents the data concerning the first hypothesis. Table 1 clearly indi-

Table 1

\begin{tabular}{|c|c|c|c|}
\hline \multicolumn{4}{|c|}{$\begin{array}{c}\text { Distribution of "stmong" responses ( } \mathbf{R t}_{1} \text { up to } 3 \mathrm{sec} \text { ) } \\
\text { according to set and reproduction }\end{array}$} \\
\hline & $\mathrm{RP}_{+}$ & RP- & $T$ \\
\hline Reproduction Set & 292 & 70 & 362 \\
\hline No Reproduction Sef & 297 & 211 & 508 \\
\hline$T$ & 589 & 281 & 870 \\
\hline
\end{tabular}


Table 2

Distribution of "weak" responses (Rt 1 above $3 \mathrm{sec}$ ) according to set and reproduction

\begin{tabular}{lccc}
\hline & RP+ & RP- & T \\
Reproduction Set & 10 & 28 & 38 \\
No Reproduction Set & 47 & 45 & 92 \\
$T$ & 57 & 73 & 130 \\
\hline
\end{tabular}

$X^{2}=6.7 ; p<0.1$

cates that reproduction set increases the chances of a strong response to be reproduced. The second hypothesis is tested in Table 2. The data give clear support to the notion that reproduction set decreases the chances of weak responses. This interference effect, however, appears to be weaker than the intensification of strong responses effect.

Since the cutoff point of $\mathrm{Rt}_{1}=3 \mathrm{sec}$ has no theoretical rationale, and it may at least partially determine the results, It was important to raise the question: Into which response strength category do the responses on which $\mathbf{R t}_{1}$ was exactly $3 \mathrm{sec}$ fit? It was found that on these responses $(N=140)$ reproduction set increased the chances of reproduction $\left(X^{2}=6.87, p<.01\right)$, and, hence, justiffed their inclusion in the category of strong responses. This may be vlewed as a type of post facto rationale.

In order to test the third hypothesis it is necessary to analyze the reaction time on $T_{2}\left(R t_{2}\right)$ in cases of Rp+. In view of the big individual differences in Rt, any such analysis should preferably be based on intraindividual comparisons. In the present study it is possible to compare $\mathrm{Rt}_{2}$ to $R \mathrm{t}_{1}$ of a particular $\mathrm{S}$ tothe same stimulus. $\mathrm{Rt}_{2}$ may be longer than, equal to, or shorter than $\mathrm{Rt}_{1}$, and the third hypothesis states that in $\mathbf{R p}+$ responses, reproduction set should reduce the Rt. Table 3 presents the results.

Table 3 strongly supports the research hypothesis, and it appears that the drive properties of reproduction set can be demonstrated in the latency index of reaction potential as well.

\section{Discussion}

The data of this study indicate that reproduction set in a word association task increases the total drive (D) level of the organism. This has differential effects upon strong and weak responses, in line with Hullian theory. The tendency of $D$ to reduce the reaction potential of weak responses was, however, found to be weaker than the intensification of strong responses. This may reflect an inadequacy of the operational
Table 3

Distribution of changes in Rt in Rp+ responses

according to set

\begin{tabular}{lcccc}
\hline & $R_{t_{2}}<R_{t_{1}}$ & $R_{t_{2}}=R_{t_{1}}$ & $R_{t_{2}}>R_{t_{1}}$ & $T$ \\
Response Set & 147 & 102 & 53 & 302 \\
No Response Set & 118 & 156 & 70 & 344 \\
$T$ & 265 & 258 & 123 & 646 \\
\hline
\end{tabular}

$X^{2}=14,1 ; d f=2 ; p<.001$

definition of response strength, since even responses with relatively long latencies were still preferred by the Ss over other responses. This suggests that while strong responses can be viewed as strong by both the probability and latency indices of reaction potential, this is not the case with weak responses. Such an asymmetry in the operational definitions makes the necessity of a thorough analysis of individual response hierarchies especially relevant.

On the other hand, the fact that the hypotheses were supported by the data allowed a post facto analysis of the cutoff point to be made, and partially justified the arbitrary choice of the latency of $3 \mathrm{sec}$ employed in this study.

Finally, it is apparent that reproduction set is an important variable which should be controlled in any attempt to compare studies in this area. The fact that the large majority of responses $(87 \%)$ fell into the category of strong responses suggests that the main effect of reproduction set is to increase the probability and reduce the latency of reproduction. A more detailed analysis, based on theoretical considerations such as the present one, however, may reach beyond the main effect.

\section{References}

Hull, C. L. A behavior system. New Haven: Yale University Press, 1952.

Moran, L. J., Mefferd, R. B., Jr., \& Kimble, J. P., Jr. Idiodynamic sets in word association. Psychol. Monogr., 1964, 78, No. 579.

Rapaport, D., Gill., M., \& Schafer, R. Diagnostic psychological testing. Vol. 2. Chicago: Year Book, 1946.

Spence, K. W., Farber, I. E., \& McFann, H. H. The relation of anxiety (Drive) level to performance in competitional and noncompetitional paired-associates learning. $J$. exp. Psychol, $1956,52,296-310$.

Taylor, J. A., \& Spence, K. W. The relationship of anxiety level to performance in serial learning. J. exp. Psychol., 1952, 44, 61-64.

\section{Note}

1. The author is greatly indebted to Mr. M. Zuckerman for his invaluable help in the collection and analysis of the data. 